te Mobilisierung und die Organisation der »Rasengerichte ${ }^{21}$ symbolisch deutlich, dass noch lange gemeinsam über die Entstehung und Verarbeitung der Katastrophe geredet und nachgedacht werden soll und dass man - im Prinzip jedenfalls - eine Kollektivschuld der Nicht-Opfer verneint. Hinter dem Versöhnungs- und Einheitsdiskurs der herrschenden politischmilitärischen Kräfte sollte man indessen sehr genau nach spezifischen Interessen (und totalitaristischer Ausbeutung des Genozidbonus) schauen. Das unbestreitbare Verdienst, einen Völkermord beendet zu haben, und die Furcht vor einem Aufleben neuer Konflikte können auf Dauer demokratische Defizite nicht entschuldigen. ${ }^{22}$ Wo - wie in jeder Gesellschaft - Konflikte schwelen, provoziert die strafrechtlich durchgesetzte Beschwörung von Einheit und Versöhnung oft das Gegenteil. Demokratie und Rechtsstaat entzaubern das »Volksmärchen« der Versöhnung, oder, wie Alain Finkielkraut es in seiner Analyse des Barbie-Prozesses für eine andere Post-Genozidphase formuliert hat:

»....doch leider behängen wir den Antinazismus mit dem totalitären Phantasma der offenen Herzen und des Glücks der Verschmelzung. Dem Trauma der homogenen Volksgemeinschaft aus Blut und Boden setzen wir die »übertriebene Nähe einer gleichmacherischen Brüderlichkeit entgegen. Ganz so als wäre nichts geschehen, als hätte keine Katastrophe unsere Epoche zu einer Epoche der Trauer gemacht, sinkt die Nacht der Idylle wieder auf uns herab. Die Liebe entthront Polemos, das Gefühl füllt die Zwischenräume

21 Die ja traditionell gerade das Gegenteil staatlicher Gerichte waren, nämlich echte grass-root settings darstellten.

22 Vgl. hierzu das Diskussionspapier von P. Uvin, »Wake up! Some Policy Proposals for the International Community in Rwanda«, June 2003 (»...improved rule of law is probably the socio-political change with the highest pay-off for Rwanda's future«). der Differenz aus und ersetzt die antagonistische Äußerung von Meinungen durch die lyrische Vereinigung von Personen...In einer scheinbaren Versöhnung mit den Idealen der Demokratie verdunkelt sich die Politik...« ${ }^{23}$

Auf die Eingangsfrage bezogen heißt das: Konflikte sind normal und bedürfen an sich keiner besonderen Behandlung in Form vom Versöhnungskampagnen. Der Rechtsstaat und die Politik sind schlicht dazu da, die friedliche Austragung dieser Konflikte zu ermöglichen. Ruanda war und ist kein »failing state«, im Gegenteil: Gerade die effektive, europäischen Vorbildern nachempfundene Verwaltungsstruktur erlaubt es heute, innerhalb von drei Tagen ein nationales Verbot des Urinierens in der Öffentlichkeit und der Benutzung von Plastiktüten zu etablieren. Diese Struktur war Voraussetzung des Genozids. Bei einem Staat, dessen Größe der Belgiens und dessen Einwohnerzahl der des OLG-Bezirkes Hamm entspricht und in dem es im Gegensatz zu vielen anderen Ländern Afrikas keine lokalen Rechtstraditionen (mehr) gibt, können mit der Etablierung landesweit wirksamer rechtsstaatlicher Strukturen die Wiederholung der Katastrophe vermieden und jedenfalls die Rahmenbedingungen für individuelle TäterOpfer-Verständigung geschaffen werden. Das Beste, was man gegenwärtig über die Gacaca-Verfahren sagen kann, ist, dass sie eine conditio sine qua non für die innerruandische Verständigung darstellen und trotz ihrer Mängel alternativlos sind. Nach den ersten Urteilen, die in etwa einem Jahr zu erwarten sind, und den Erfahrungen mit weiteren vorläufigen Freilassungen bis dahin wird neu darüber nachzudenken sein.

23 A. Finkielkraut, »Die vergebliche Erinnerung - Verbrechen gegen die Menschheit«, Berlin 1989, S. 87.

\title{
Bundeswehr-Modell »2025«
}

\section{Kommission »Europäische Sicherheit und Zukunft der Bundeswehr«am IFSH}

\section{Friedenspolitische Grundprämisse}

Jede europäische Gemeinsame Außen- und Sicherheitspolitik muss zuerst und vor allem völkerrechtlich sanktionierte Friedenspolitik, eine Politik der zivilen Instrumente sein. Dem Primat der zivilen Krisenprävention ist durch eine signifikante Umschichtung der Mittel zugunsten nichtmilitärischer Sicherheitskräfte, die für die Gefahren und Risiken der Zukunft von größerer Relevanz sind, Rechnung zu tragen. Nur damit kann das Leitbild der Europäischen Union als »Friedensmacht» Glaubwürdigkeit gewinnen.

\section{Gesellschaftspolitische Prämissen}

2.1 In Europa existiert seit geraumer Zeit ein eindeutiger Trend weg von der allgemeinen Wehrpflicht, hin zu Freiwilligenstreitkräften. Auch die Bundeswehr praktiziert längst keine allgemeine Wehrpflicht mehr, sondern nur noch eine Art Fassadenwehrpflicht: Wenn von 250.000 Soldatinnen und Soldaten nur noch 30.000, also zwölf Prozent, echte Grundwehrdienstleistende sein sollen, kann nicht mehr ernsthaft von Wehrpflichtarmee gesprochen werden. 220.000 Militärangehörige werden zukünftig Freiwillige - Freiwillig zusätzlichen Wehrdienst 
Leistende (FWDL), Soldatinnen und Soldaten auf Zeit sowie Berufssoldatinnen und Berufssoldaten - sein. Sicherheitspolitische Gründe werden für die zwangsweise Besetzung dieser 30.000 Wehrpflichtigenplätze schon lange nicht mehr angeführt. Auch alle anderen Argumente, die für die Beibehaltung der Wehrpflicht genannt werden, relativieren sich, wenn der Wehrpflichtanteil nur noch zwölf Prozent beträgt. Diese Plätze können genauso gut mit Freiwilligen besetzt werden. Wehrpflicht ist nicht mehr nötig - auch nicht als Fassade. Ihre Abschaffung ist überreif und überhaupt kein Schaden für die Innere Führung.

2.2 Es ist jedoch sicherzustellen, dass die künftigen deutschen Freiwilligenstreitkräfte auch weiterhin in mindestens demselben Ausmaß gesellschaftlich integriert bleiben, wie es derzeit der Fall ist. Ein zentrales Kriterium dafür ist der ständige personelle Austausch zwischen Militär und zivilem Umfeld. Dazu könnten nicht zuletzt eine drastische Reduzierung des Berufssoldatenanteils sowie eine auf ein vertretbares Mindestmaß begrenzte Dienstzeit der übrigen Freiwilligen beitragen.

2.3 Die Chance zur inneren Demokratisierung der Bundeswehr muss weit besser als bisher genutzt werden - nicht zuletzt auch, um den Dienst in den Streitkräften attraktiver zu machen. Denn zumindest außerhalb der Einsätze ließen sich die internen militärischen Strukturen und Verfahren sehr weitgehend demokratisieren bzw. an demokratischen Normen und Werten ausrichten. Eventuelle Beschränkungen hinsichtlich der Demokratisierung der Bundeswehr dürfen einzig und allein mit den wirklich unumgänglichen funktionalen Erfordernissen begründet werden.

2.4 Die Bundeswehr ist ein »Parlamentsheer « - und muss es ohne Abstriche bleiben. Der Parlamentsvorbehalt bei Auslandseinsätzen ist mitnichten ein lästiges Hindernis, sondern einer entwickelten Demokratie nur angemessen. Die Einführung gesetzlicher Regelungen, die keine substanzielle Stärkung der parlamentarischen Mitwirkungsrechte, sondern deren Aufweichung darstellen, ist abzulehnen.

2.5 Die gegenwärtigen Bemühungen zum Aufbau und zur Stärkung der sicherheitspolitischen Handlungsfähigkeit der Europäischen Union und die Installierung entsprechender Mechanismen und Kapazitäten könnten einen zusätzlichen Faktor zur Aushebelung nationaler Parlamentsvorbehalte darstellen. Grundsätzlich ist daher für bewaffnete Streitkräfteeinsätze im Rahmen der Europäischen Sicherheits- und Verteidigungspolitik $z u$ sätzlich die Zustimmung des Europäischen Parlaments $\mathrm{zu}$ fordern.

\section{Militärpolitische Prämissen}

3.1 Schnittstellen zwischen dem Auftrag militärischer und polizeilicher Kräfte beim Einsatz in Krisenregionen sind klarer und eindeutiger zu definieren und das Zusammenwirken aller sicherheitspolitischer Instrumente zu optimieren. Polizeiliche Aufgaben können auch künftig allenfalls zeitlich befristeter Teil des Auftragsspektrums der Streitkräfte sein.

3.2 Der gegenwärtige Trend zum Aufbau multinationaler Streitkräftestrukturen und zur Arbeitsteilung unter Partnern und Verbündeten könnte seine Grenzen darin fin- den, dass nationale Entscheidungen zur Nichtteilnahme an bestimmten Einsätzen zumindest noch für eine längere Übergangszeit möglich bleiben sollten, ohne die europäische Handlungsfähigkeit insgesamt in Frage zu stellen.

3.3 Nicht jeder militärische Einsatz mit europäischer bzw. deutscher Beteiligung muss als Langzeitmission angelegt sein. Wenn also bereits der Einsatz bewaffneter Streitkräfte der absolute Ausnahmefall sein soll, dann sind Langzeitmissionen gleichsam nur der Ausnahmefall im Ausnahmefall. Ohnehin kann der Einsatz von Streitkräften in Krisenregionen auf Dauer politische Lösungen nicht ersetzen. Auf jeden Fall ist vor jedem Einsatz dessen politisches Ziel zu definieren und auf dessen Grundlage Art, Umfang und Zusammensetzung der verschiedenen sicherheitspolitischen Kräfte und Mittel festzusetzen.

3.4 Nicht jede Langzeitmission, sofern sie überhaupt erforderlich ist, muss unter permanenter Beteiligung von Kontingenten ein und derselben Nation erfolgen. In jedem Fall sollte im Rahmen einer künftigen ESVP ein - entsprechend der wachsenden Anzahl ihrer Teilnehmerstaaten - strukturiertes Rotationsverfahren entwickelt werden.

3.5 Der legitimierte gewaltsame Einsatz militärischer Macht schließlich, etwa zur Ausschaltung einer unmittelbaren Bedrohung oder Verhinderung eines Genozids, muss in jeder Hinsicht das äußerste Mittel innerhalb des sicherheitspolitischen Instrumentariums bleiben. Die speziell für derartige Aufgaben ausgebildeten und ausgerüsteten Kräfte können daher besonders engen strukturellen Begrenzungen unterliegen.

\section{Strukturelle Grundlinien der Bundeswehr}

4.1 Die hinsichtlich Auftrag, Ausbildung und Ausrüstung neu vorgenommene Unterteilung der deutschen Einsatzstreitkräfte in Eingreif- und Stabilisierungskräfte erscheint künftigen sicherheitspolitischen Risiken und Gefahren grundsätzlich angemessen. Jedoch sollte das geplante Stärkeverhältnis zwischen beiden Kräftekategorien zugunsten der Stabilisierungskräfte modifiziert werden. Unter Zugrundelegung des im »Bundeswehrmodell $200 F$ « vorgeschlagenen Rahmens von zwölf Einsatzbrigaden sollten daher lediglich zwei Brigaden den Eingreifkräften, dagegen zehn den Stabilisierungskräften zugeordnet werden.

4.2 Zusätzlich sollten Kräfte für zwei binationale Brigaden bereitgehalten werden: Neben der deutsch-französischen ist auch die Aufstellung einer deutsch-polnischen Brigade vorzusehen. Grundsätzlich berechtigte Einwände über Effizienzdefizite multinationaler Brigaden sollten in diesem Falle zugunsten der überragenden politisch-symbolischen Bedeutung zurückgestellt werden.

4.3 Die Einsatzkräfte werden durch Luft- und Seestreitkräfte komplettiert. Diese sollten jeweils drei Geschwaderäquivalente nicht überschreiten. Insgesamt umfassen die Einsatzkräfte demnach ca. 51.000 Soldatinnen und Soldaten.

4.4 Die Teilstreitkräfte Heer, Luftwaffe und Marine sollten nicht nur einen Teil ihrer bisherigen Aufgaben (an die neu eingerichteten Organisationsbereiche Streitkräftebasis und Sanitätsdienst) abgeben, sondern gänzlich ver- 
schmolzen werden. Alle bestehenden Parallelstrukturen sind aufzuheben. Für die gesamten Streitkräfte genügt beispielsweise eine einzige Offizierschule. Ebenso könnten sämtliche Einsatzkräfte ständig einem gemeinsamen Führungskommando unterstellt werden. Daneben sind nur ein zentrales Kommando für die Ausbildung in den Streitkräften sowie ein zentrales Kommando für Führungs-, Kampf- und Einsatzunterstützung vorzusehen. Diese drei Kommandobehörden werden direkt dem Generalinspekteur der Bundeswehr unterstellt. In dessen Stab fungieren die Inspekteure von Luftwaffe und Marine demzufolge nur noch als eine Art herausgehobene Inspizienten. Ein Inspekteur des Heeres indessen ist überflüssig.

4.5 Die militärischen und zivilen Bürokratien in der Bundeswehr außerhalb der Einsatzkräfte können größtenteils abgebaut werden. Denn beim bisherigen Transformationsprozess verringerten sich zwar der Personalumfang und die Anzahl der Verbände, die Anzahl der Hierarchieebenen aber blieb erhalten. Die Leitungsspannen, d.h. die Zahl der direkt unterstellten Truppenteile bzw. Dienststellen, nahmen daher - vor allem auf den oberen Ebenen - zum Teil deutlich ab. Fast könnte man den Eindruck gewinnen, die Strukturen der Streitkräfte leiten sich weniger aus ihrem Auftrag als aus dem Bestreben ab, möglichst viele gut dotierte Dienstposten zu erhalten. Der Inspekteur des Heeres etwa, dem früher drei Korps, drei Territorialkommandos und das Heeresamt, also insgesamt sieben Höhere Kommandobehörden, direkt unterstellt waren, »führt « heute - mit einem aufgeblähten Stab - nur noch deren zwei (Heeresführungskommando und Heeresamt). Die Folgerung liegt nahe, er sei entweder früher überfordert gewesen oder heute unterfordert oder beides.

4.6 Der künftige Personalumfang der Streitkräfte kann unter diesen strukturellen Vorgaben langfristig auf bis zu 125.000 Soldatinnen und Soldaten reduziert werden.

4.7 Die damit eingesparten Personalkosten würden eine signifikante Erhöhung der Investitionsquote im Vertei- digungshaushalt ermöglichen. Dennoch ließe sich bis zum Ende der Reduzierungsphase der Wehretat auf ca. 16 Mrd. Euro absenken.

\section{Die europäische Perspektive}

5.1 Das Ziel einer Europäischen Armee ist allenfalls langfristig erreichbar. Auch einer vollständigen Harmonisierung der nationalen Streitkräfte stehen noch viele, teilweise unüberwindlich erscheinende Hindernisse entgegen. Aber bereits heute könnten die Armeen der einzelnen EU-Staaten hinsichtlich ihrer Attraktivität in eine Art Wettbewerb treten und in ganz Europa um ihr Personal werben. Die Bundeswehr sollte also zumindest für alle EU-Bürgerinnen und -Bürger geöffnet werden.

5.2 Die Pläne zum Aufbau einer eigenen militärischen Infrastruktur der EU müssen mit dem Leitbild der »Friedensmacht Europa « in Einklang stehen. Neben dem bestehenden EUROKORPS könnten zwar - in eng begrenzter Zahl - weitere integrierte Führungsstäbe (»II. Europäisches Korps« usw.) aufgestellt werden, denen jedoch auch die gemäß »European Headline Goal« beschlossenen, schnell verlegbaren militärischen Einsatzkräfte und die so genannten »Einsatzgruppen« (»Battle Groups «) für eventuelle Einsätze zu unterstellen wären.

5.3 Während bei den Ausbildungseinrichtungen der Streitkräfte im EU-Rahmen eine Arbeitsteilung zwischen den einzelnen Nationen in weit stärkerem Maße als bisher erfolgen kann, liegen die diesbezüglichen Grenzen bei den Einsatz- sowie den Unterstützungskräften wohl wesentlich enger: Die nationale Option zur Nichtbeteiligung an einem militärischen Einsatz soll ja erhalten bleiben. Unzulänglichkeiten in der militärischen Handlungsfähigkeit der EU mögen ein sicherheitspolitisches Handicap darstellen - ein Verzicht auf die Vision von der »Friedensmacht« hingegen wäre ein Unglück. 